phys. stat. sol. (a) 175, 289 (1999)

Subject classification: 78.66.Fd; 72.20.Ht; 72.30.+q; 72.40.+w; 73.40.Gk; S7.12

\title{
Near-Zone Field Effect of FIR Laser Radiation on Tunnel Current through the Schottky Barrier under Plasma Reflection Condition
}

\author{
A. Ya. Shulman ${ }^{1}$ ) (a), S. D. Ganichev (b), I. N. Kotelnikov (a), E. M. Dizhur (c), \\ W. Prettl (b), A. B. Ormont (a), Yu. V. Fedorov (a), and E. Zepezauer (b)
}

(a) Institute of Radioengineering and Electronics, Russian Academy of Sciences, 103907 Moscow, Russia

(b) Institut für Experimentelle und Angewandte Physik, Universität Regensburg, D-93040 Regensburg, Germany

(c) Institute for High Pressure Physics, Russian Academy of Sciences, 142092 Troitsk, Russia

(Received May 8, 1999)

Far infrared (FIR) radiation of high-power pulsed laser incident normal to the surface of GaAs/ metal tunnel structures with a self-consistent Schottky barrier gives rise to a change in the tunnel conductance. It has been shown that the observed photoresistive effects are caused by ponderomotive forces of the radiation field on the free electron plasma in the junctions. The change of tunnel conductance rises linearly with increasing intensity at low power levels and proceeds into a strongly superlinear dependence at high intensities. It is shown that this superlinearity is a result of an enhancement of the local radiation field in the near zone of diffraction by inhomogeneities at the metal-semiconductor interface and depends strongly on the roughness of the metal electrode. Experimental results are compared to a nonlinear extension of the theory of electron redistribution due to the radiation pressure.

\section{Introduction}

The conductance of tunnel Schottky junctions formed by highly doped n-GaAs and a semitransparent metal electrode on its surface is changed by normally incident electromagnetic radiation with frequency in the far infrared below the plasma edge of the electron gas in GaAs. The electromagnetic wave being totally reflected by the plasma transfers momentum to the electrons of the semiconductor. This leads to a spatial redistribution of the electrons due to the radiation pressure and yields a photoresistive effect resulting from the corresponding change in the shape of the self-consistent Schottky-barrier [ 1 to 3]. The change of tunnel conductance in response to pulsed far-infrared (FIR) radiation rises linearly with increasing intensity at low power levels and proceeds into a strongly superlinear dependence at high intensities. This superlinearity is a result of an enhancement of the local radiation field in the near-zone owing to the diffraction by inhomogeneities at the metal/semiconductor interface. Because of the high doping level the width of the Schottky barrier is of the order of 20 to $30 \mathrm{~nm}$ which is much less

1) e-mail: ash@cplire.ru; Fax: (7-095) 203-8414; Tel.: (7-095) 203-4987 
than the extent of near-zone field variations of the order of $\mu \mathrm{m}$. Therefore, the field strength in the Schottky barrier region is to be of the same order as at the inhomogeneities themselves and the onset of the nonlinear response depends essentially on the grade of the surface inhomogeneity of the metal electrodes.

Here we present experimental results for $n-G a A s / A l$ tunnel junctions and compare them to a nonlinear extension of the theory of electron redistribution due to radiation pressure. Both theory and experiment yield an effective enhancement of the radiation intensity close to the semiconductor/metal-gate interface compared to the incident plane wave up to about $10^{5}$.

\section{Theory}

\subsection{Reconstruction of the Schottky barrier}

The equation of motion of a degenerate electron plasma in the semiconductor under the action of the electrostatic barrier field $\mathbf{E}_{\mathrm{st}}$ and an electromagnetic wave field $\left(\mathbf{E}_{\mathbf{1}}, \mathbf{B}_{\mathbf{1}}\right)$ can be written as

$$
m n(x) \frac{\mathrm{d} \mathbf{v}}{\mathrm{d} t}=-\nabla p(x)-n(x) e \mathbf{E}(x)-n(x) \frac{e}{c}\left(\mathbf{v} \times \mathbf{B}_{1}\right)-m n(x) \frac{\mathbf{v}}{\tau} .
$$

Here $\mathbf{E}(x)=\mathbf{E}_{\mathrm{st}}(x)+\mathbf{E}_{1}(x, t)$ is the electric field, $\mathbf{B}_{1}$ is the magnetic field, $p(x)$ is the pressure of the electron plasma with density $n(x)$ and $\mathbf{v}(x, t)$ is the electron drift velocity. The incident radiation is directed along the $x$-axis normally to the semiconductor surface.

The time-averaged Lorentz force acting on the electrons may be represented as ponderomotive force originated by the divergence of the Maxwell stress tensor

$$
F_{i}=\frac{\mathrm{d} T_{i k}}{\mathrm{~d} x_{k}}, \quad \text { where } \quad T_{x x}=\frac{1}{8 \pi}\left(\kappa E_{\mathrm{st}}^{2}-\frac{\kappa}{2}\left|E_{1}^{2}\right|-\frac{1}{2}\left|B_{1}^{2}\right|\right)
$$

and $\kappa$ is the dielectric constant of the GaAs lattice. The equilibrium condition of the plasma elemental volume is $\mathrm{d} \mathbf{v} / \mathrm{d} t=0$, that is

$$
-\frac{\mathrm{d}}{\mathrm{d} x} p-e N E_{\mathrm{st}}(x)+\frac{\mathrm{d}}{\mathrm{d} x} T_{x x}=0 .
$$

The integration of this relation gives us the equation for the electrostatic field of the barrier in the depletion layer with account for the radiation

$$
\frac{\kappa}{8 \pi} E_{\mathrm{st}}^{2}=N \Phi(x)-\frac{2}{5} \mu+\frac{\kappa}{16 \pi}\left(\frac{\omega_{\mathrm{p}}}{\omega}\right)^{2}\left|E_{1}^{2}\right| .
$$

Here $\Phi$ is the electrostatic barrier potential, $\mu$ is the Fermi energy of the semiconductor electron plasma, $N$ is the ionized donor concentration in the semiconductor, $\omega_{\mathrm{p}}$ is the plasma frequency, $\omega$ is the frequency of the incident radiation.

\subsection{Change in the tunnel current}

The tunnel current of the irradiated junction may be presented in a simplified form

$$
I^{*}\left(V^{*}, T^{*}, J\right) \propto \int_{0}^{\infty} \mathrm{d} \varepsilon\left[f\left(\varepsilon, T^{*}\right)-f\left(\varepsilon+e V^{*}, T\right)\right] \exp \left[-G^{*}\left(\varepsilon, V^{*},\left|E_{1}\right|^{2}\right)\right] .
$$




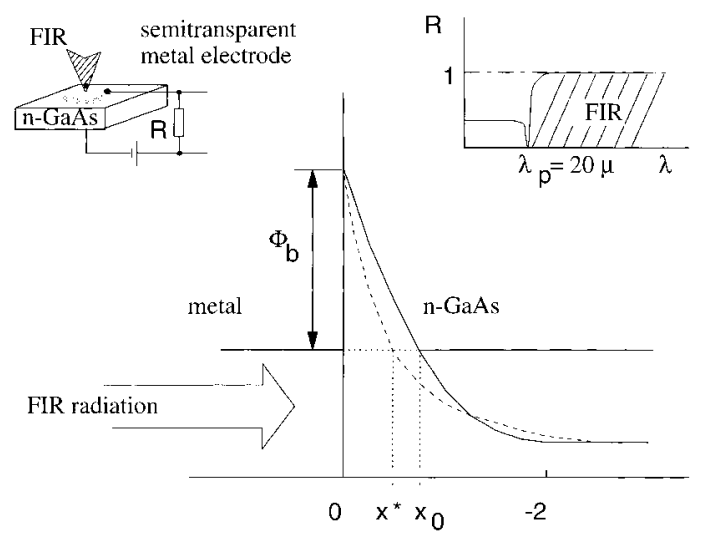

Fig. 1. Schematic representation of the reconstruction of the Schottky barrier due to the incident FIR radiation. The dotted line represents the reconstructed barrier. Insets: Experimental set-up using a standard photoconductivity measurement circuit (left). Reflectivity of the free electron gas in $\mathrm{n}$-GaAs showing the plasma reflection edge (right)

Here $f(\varepsilon, T)$ is the Fermi distribution function of the electrons with temperature $T, \varepsilon$ is the electron energy, $V$ is the bias voltage without irradiation, $V^{*}=V-\Delta U_{\mathrm{L}}$, $\Delta U_{\mathrm{L}}=U_{\mathrm{L}}^{*}-U_{\mathrm{L}}$ is the change in the voltage drop on the load resistance (see Fig. 1). The "*" denotes the values of the correponding quantities during irradiation. The electron temperature $T^{*}$ takes into account possible electron heating in the radiation field. The quasi-classical expression for the barrier transparency now depends on the electromagnetic wave amplitude and may be written as

$$
G^{*}\left(\varepsilon, V,\left|E_{1}\right|^{2}\right)=\frac{2 \mu}{\hbar \omega_{\mathrm{p}}} \int_{\varepsilon}^{\Phi_{\mathrm{b}}} \mathrm{d} \Phi \sqrt{\frac{\Phi-\varepsilon}{\Phi-\frac{2}{5}+K_{\mathrm{e}} \frac{u}{\mu}}} .
$$

Here $\Phi_{\mathrm{b}}(V)$ is the band-bending height at the semiconductor-metal interface, $u$ is the high-frequency potential related to the intensity $J$ of the incident radiation,

$$
u=\frac{e^{2}\left|E_{1}\right|^{2}}{4 m \omega^{2}}=\frac{J}{N c} .
$$

The coefficient $K_{\mathrm{e}}$ describes the electric field enhancement at the semiconductor surface. The change in the tunnel current due to incident radiation is

$$
\Delta I\left(V^{*}, T^{*}, J\right)=I^{*}\left(V^{*}, T^{*}, J\right)-I(V, T, J=0) .
$$

A quantitative description of the near-zone field effect needs to introduce an effective intensity $J_{\mathrm{e}}=K_{\mathrm{e}} J$ and to take into account that only a small fraction $\eta$ of the junction area is affected by the enhanced near-zone field. Thus, we obtain for the total current response

$$
\Delta I_{\mathrm{t}}=\eta \Delta I\left(V^{*}, T^{*}, J_{\mathrm{e}}\right)+(1-\eta) \Delta I\left(V^{*}, T, J=0\right) .
$$

The important feature of Eq. (5) should be stressed. The two terms on the right-hand side have opposite signs since the barrier transparency and the electron temperature are increased in the small region of high near-zone field while the current through the remaining area is decreased owing to a drop in the bias during the laser pulse if $R_{\mathrm{L}} \neq 0$. As a result, at high intensities the observed response is formed by a delicate 
balance between these two terms giving rise to a rather high sensitivity of the numerical analysis of the experimental data to inconsistence of the theoretical model with real physics of the phenomenon.

And finally, the equation for the response $\Delta U_{\mathrm{L}}=R_{\mathrm{L}} \Delta I$ in the measurement circuit,

$$
R_{\mathrm{L}} \Delta I\left(V^{*}, T^{*}, J\right)+V^{*}-V=0 .
$$

Another important variable in Eq. (6) is the electron temperature $T^{*}$ which is calculated in the electron temperature approximation with taking into account the electron momentum loss due to ionized impurities and the energy loss due to nonequilibrium LO and intervalley $(\Gamma \leftrightarrow \mathrm{L})$ phonons. Taking the approach $[5,6]$ to the electron-phonon heating of the dependence of the electron temperature $T^{*}$ on the intensity $J$ was derived in the following form

$$
T^{*}=\hbar \omega_{0} / \ln \left(1+\frac{1}{2 \frac{u_{\mathrm{e}}}{\hbar \omega_{0}} \frac{\tau_{\varepsilon}}{\tau}+N^{0}\left(\omega_{0}, T\right)}\right),
$$

where $\omega_{0}$ is the phonon frequency, $N^{0}$ is the equilibrium Bose distribution, $u_{\mathrm{e}}=u\left(J_{\mathrm{e}}\right)$ (see Eq. (3)), $\tau_{\varepsilon}$ and $\tau$ are the energy and momentum relaxation time of the electron gas, respectively. The expression Eq. (7) is derived in high frequency approximation $\omega \tau>1$. The ratio of the relaxation times $\tau_{\varepsilon} / \tau=a$ was chosen as one of the fitting parameters being independent of $J$ in addition to the $K_{\mathrm{e}}$ and $\eta$ characterizing the electrodynamic part of the problem.

Since the phonon heating is also present in the system the increase of the electron temperature in L-valley is to be taken into consideration. This was done by introducing one more parameter $b$ by means of the relation $\Delta T_{\mathrm{L}}^{*}=b \Delta T_{\Gamma}^{*}$.

\section{Experimental Results}

Tunnel semiconductor/metal Schottky junctions have been prepared by evaporation under various conditions of aluminum on MBE grown n-doped GaAs (2) with $2 \times 10^{18} \mathrm{~cm}^{-3}$ donor concentration. Special efforts were made to obtain semitransparent metal electrodes with different grades of inhomogeneity. Scanning electron micrographs of a smooth metal film (type A) and a rough film (type B) are shown in Fig. 2. An
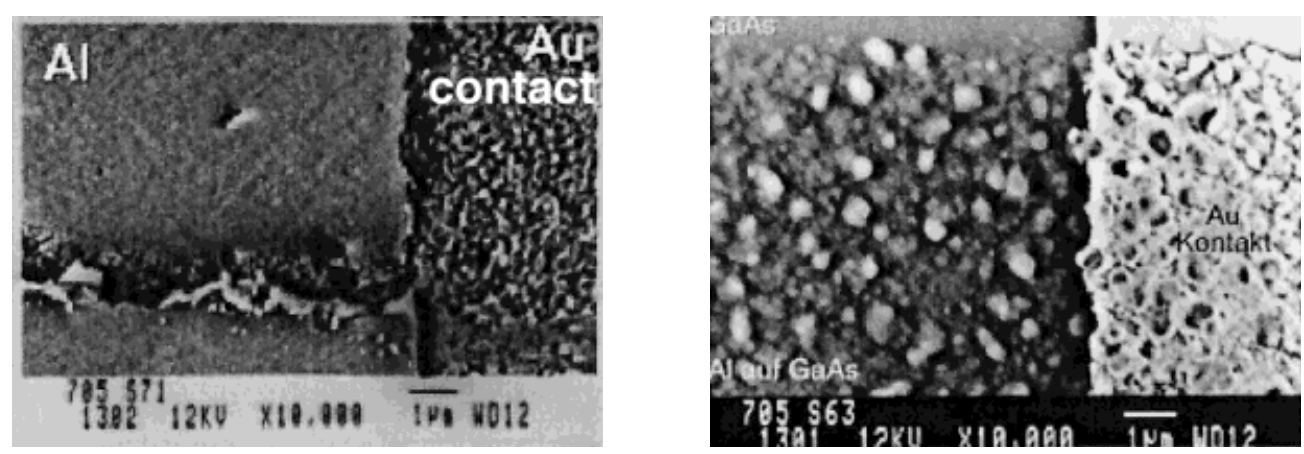

Fig. 2. Scanning electron micrographs of the metal films of Schottky-barrier junctions for various film morphologies. The smooth film is left (type A) and the rough film is right (type B) 

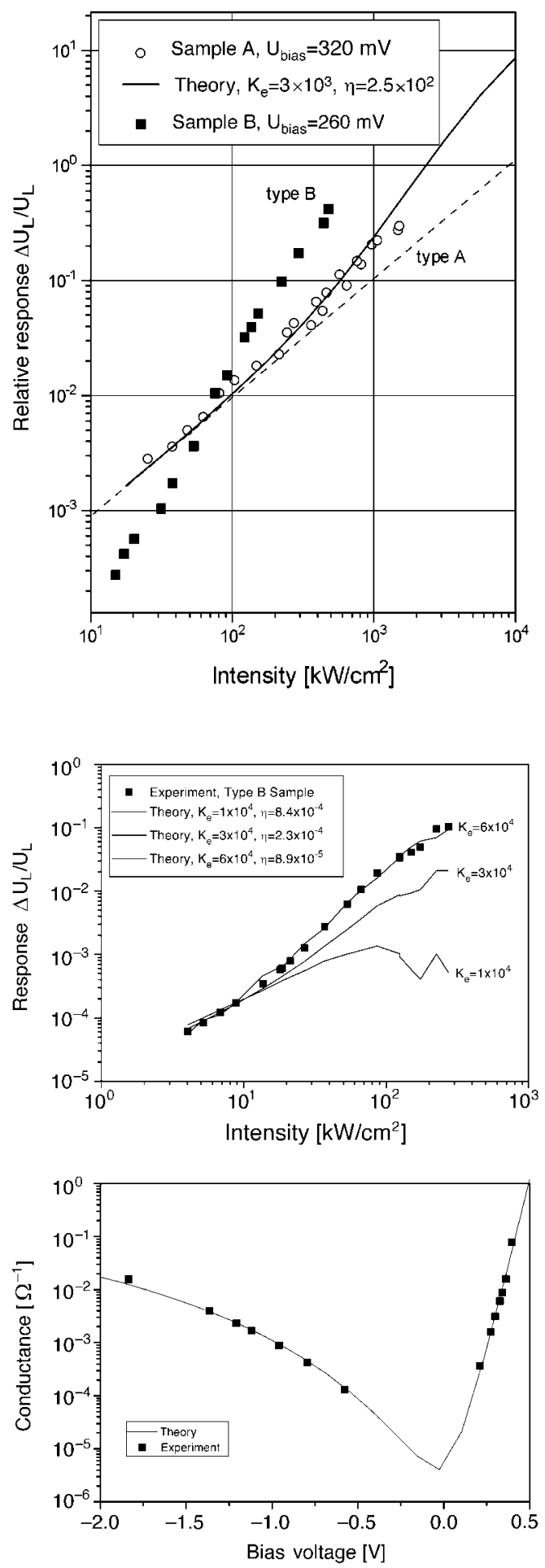

Fig. 3. Intensity dependence of the response for two kinds of tunnel junctions. Data for type A and B samples correspond to low and high near-zone enhancement of the radiation field, respectively. The dashed line shows the linear function of the intensity. The full line represents the theoretical curve calculated according to Eq. (5) at $T^{*}=T$ and $V^{*}=V$

additional important condition for the work is that the prepared metal-semiconductor structures are in fact tunneling junctions. This has been proved by measurements of tunneling characteristics by means of tunneling spectroscopy technique.

The experimental set-up of the photoconductivity measurements is sketched in Fig. 1. Pulses of a high-power FIR molecular laser [7] have been applied with $40 \mathrm{~ns}$ duration and up to $2 \mathrm{MW} / \mathrm{cm}^{2}$ peak intensity. The experimental results presented here were obtained at $90 \mu \mathrm{m}$

Fig. 4. Upper part: response of rough electrode tunnel junction (type B) with high near-field enhancement at positive bias. Squares are measurements, full lines represent theoretical curves calculated according to Eq. (5) at $T^{*}=T$ for different values of the enhancement coefficient $K_{\mathrm{e}}$. In the calculated response at low intensities and small $K_{\mathrm{e}}$ oscillations occur which are due to the interplay between increase of barrier transparancy and drop of bias during the laser pulse as discussed after Eq. (5). Lower part: Measured and calculated conductance of one of the tunnel n-GaAs/Al junctions. Fitting the calculation to experimental data has been used to determine junction parameters like the height of the Schottky barrier and the ionized impurity concentration. These parameters are essential for calculating the nearfield enhancement shown in the upper part 
wavelength using $\mathrm{NH}_{3}$ as laser active medium. The intensity incident on the samples has been controlled by calibrated teflon attenuators. The measurements were carried out at room temperature and at liquid nitrogen temperature.

Fig. 3. shows the different degrees of nonlinearity of the photoresistive response of type A and type B samples obtained at room temperature. The onset of superlinearity occurs for the sample with smooth electrodes (type A) at about $500 \mathrm{~kW} / \mathrm{cm}^{2}$ whereas the response of the other sample type with rough electrodes (type B) is superlinear in the whole intensity range of this measurement. The deviation of linear response in agreement to the measurement requires a field enhancement parameter $K_{\mathrm{e}}=10^{3}$ and an active area fraction $\eta=2.5 \times 10^{-2}$ resulting in the full line in this figure.

The experimental data for the rough electrode junction (type B) are replotted in Fig. 4, lower part, and compared to fittings using various intensity enhancement factors $K_{\mathrm{e}}$ and active area fractions $\eta$. An agreement to the extremely large nonlinearity of the measured results is obtained with an effective enhancement of the local intensity as high as $6 \times 10^{4}$. An incident $90 \mu \mathrm{m}$ radiation intensity of the order of $1 \mathrm{MW} / \mathrm{cm}^{2}$, corresponding to $18 \mathrm{kV} / \mathrm{cm}$ electric field amplitude, may lead at enhancement coefficient $K_{\mathrm{e}}$ equal to $10^{5}$ to a locally enhanced field as high as $1.6 \mathrm{MV} / \mathrm{cm}$ on a spatial scale of the order of $1 \mu \mathrm{m}$. Under plasma reflection condition, the respective amplitude of the lateral electric field inside the Schottky barrier is about $75 \mathrm{kV} / \mathrm{cm}$.

We would like to note that this is the first observation of a giant radiation field surface enhancement in the far-infrared region whose values are comparable to those observed in the visible region for giant surface Raman scattering or surface second harmonic generation. As a result, theoretical models explaining the surface enhancement by plasma resonance effects in the metal film should be questioned because the characteristic frequencies of the plasma effects of well-conducting metals lie in the UV region and cannot be responsible for an effect in the FIR region.
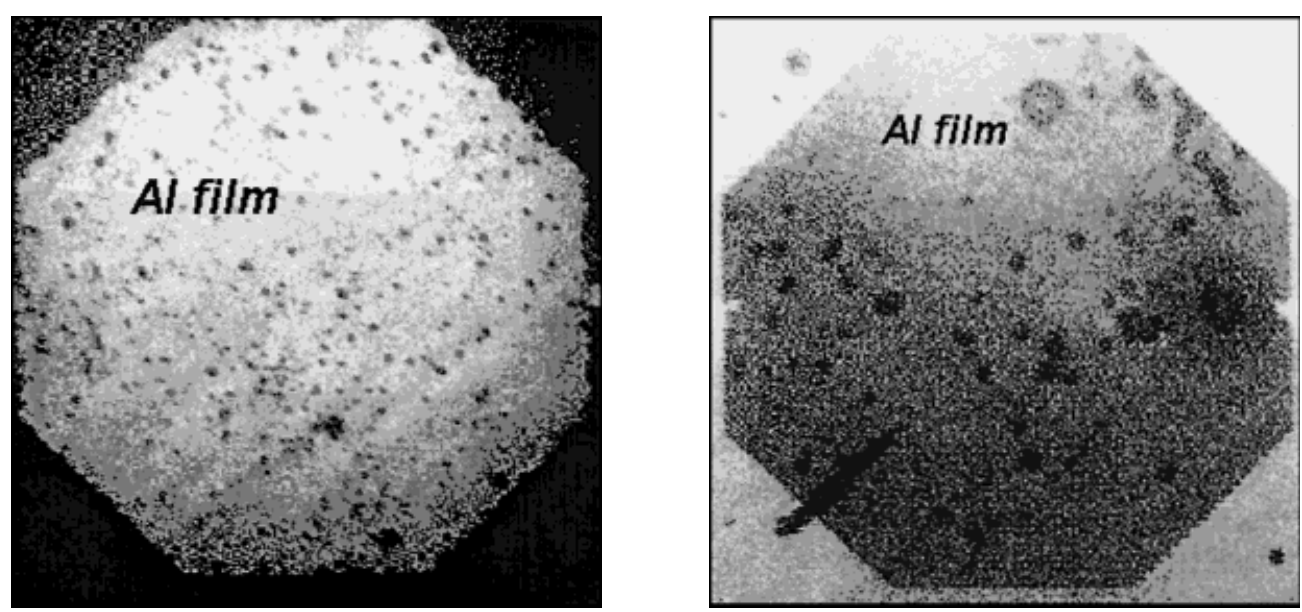

Fig. 5. Images of $\mathrm{Al}$ electrodes on GaAs surface for two subtypes of type B samples (thick metal film) with lower (left part) and higher (right part) grade of film inhomogeneity. The images are obtained by means of a $\mathrm{He}-\mathrm{Ne}$ laser scanning microscope. The diameter of each electrode is about $750 \mu \mathrm{m}$ 
The strong superlinearity of the response leads to a large radiation-induced change of the sample voltage which is comparable to the bias voltage. As the current-voltage characteristics is also strongly nonlinear, the evaluation of the photoconductivity data needs a very accurate knowledge of the current-voltage relation in the dark and at irradiation. A numerical method to calculate such a characteristic has been developed yielding good agreement to the measurements displayed in Fig. 4, upper part. These calculations were used for the exact determination of the junction parameters like barrier height and doping level since the calculated response turned out to be very sensitive on these parameters.

In order to explain the enhancement of the local electromagnetic field and the nonlinear response of the junctions, a new approach to the diffraction theory for a conducting screen with aperture has been suggested [8]. This procedure allows to carry out a qualitative or a semiquantitative evaluation of the near-zone field without cumbersome numerical solutions of integral equations. The simple calculations predict the field enhancement in a small aperture of order of $\lambda / \delta$, where $\lambda$ is the radiation wavelength and $\delta$ is the skin depth of metal film. For $\lambda \approx 10^{-2} \mathrm{~cm}$ and $\delta \approx 5 \times 10^{-6} \mathrm{~cm}$ this gives a field enhancement of about $10^{3}$ and a corresponding effective intensity enhancement of about $10^{6}$ experimentally observed here. Moreover, this approach allows to suggest an explanation of the observed dependence of the enhancement on the metal film thickness. It turned out that the responses of junctions formed by thick (but semitransparent!) metal films (type B) with two subtypes with lower (Fig. 5 left part) and higher (Fig. 5, right part) grade of film inhomogeneity have similar strong nonlinearity (compare Figs. 4 and 6). The thickness of the metal electrode appears quite essential for the
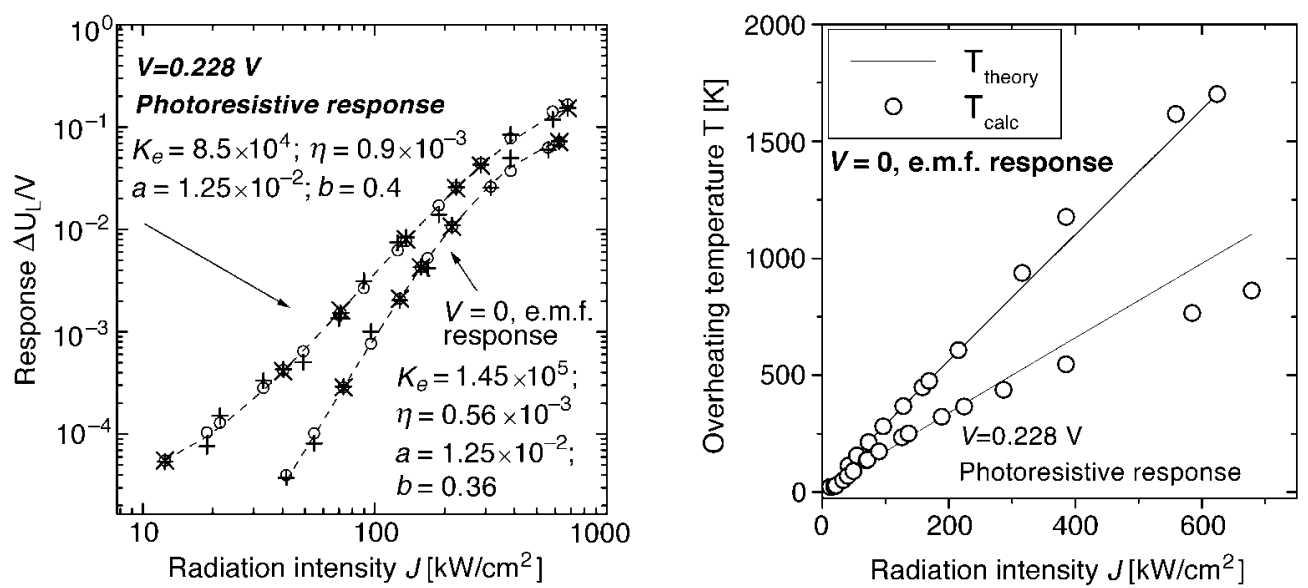

Fig. 6. Left part: photoresistive response at low bias and photo-e.m.f. as a function of intensity. + are the measured data, $x$ denote the data points selected for the least-square search of optimal parameters $K_{\mathrm{e}}, \eta, a, b, \bigcirc$ represent the calculated response obtained by numerical solution of the circuit equation Eq. (6) for the bias voltage $V^{*}$ with using the optimal parameters. The dashed line shows the smooth of the initial data set. Right part: temperature of $\Gamma$-valley electrons as a function of the incident radiation intensity determined from photoresistive response at low bias and photo e.m.f. The solid line is calculated from the theoretical formula Eq. (7) and circles ( $\bigcirc)$ again represent the solution of the circuit equation Eq. (6) for the hot electron temperature with optimal values $K_{\mathrm{e}}, \eta, b$ 
enhancement of near field. The enhancement increases with increasing film thickness as long as the film thickness is less than the skin depth of the radiation field.

In all experimental situations described until now electron heating is not important. In samples with small field enhancement, like type A, electron heating has not been observed [3]. In junctions of type B with very large field enhancement the effect of electron heating on the response is negligible at high bias voltages. However, at low bias voltages or without bias voltage electron gas heating in the surface layer of the semiconductor gets important in rough electrode samples (type B) with very high field enhancement. The electron heating is causing an e.m.f. and affects also the photoconductive signal at low and medium biases. The e.m.f. as a function of intensity is presented in Fig. 6 together with results of numerical calculations of tunneling of heated electrons across the barrier which is modified by the ponderomotive force of the nearzone field. As a result, it was found that heating of electrons not only in the $\Gamma$-valley but also in the L-valley must be taken into account as well as the generation of nonequilibrium LO phonons [5]. From this analysis the magnitude of the effective enhancement coefficient of the radiation intensity was confirmed and the temperatures of hot electrons have been determined (Fig. 6), being as high as $\approx 2000 \mathrm{~K}$ at the maximum incident intensity of the order of $1 \mathrm{MW} / \mathrm{cm}^{2}$.

Acknowledgements Financial support by Russian Foundation for Basic Researches and Deutsche Forschungsgemeinschaft is gratefully acknowledged.

\section{References}

[1] S. D. Ganichev, K. Gloukh, I. N. Kotelnikov, N. A. Mordovets, A. Ya. Shulman, and I. D. YARoshetskir; Soviet Phys. - J. Exper. Theor. Phys. 75, 495 (1992).

[2] A. Ya. Shulman, Proc. Internat. Semicond. Dev. Res. Symp., 1995, Vol. 1 (p. 229).

[3] S. D. Ganichev, A. Ya. Shulman, I. N. Kotelnikov, N. A. Mordovets, and W. Prettl, Internat. J. Infrared and Millimeter Waves 17, 1353 (1996).

[4] A. Ya. Shulman, I. N. Kotelnikov, S. D. Ganichev, E. M. Dizhur, A. B. Ormont, E. Zepezauer, and W. PretTL, Proc. 24th Internat. Conf. Physics of Semiconductors, Jerusalem 1998.

[5] I. N. Kotelnikov, A. Ya. Shulman, S. D. Ganichev, N. A. Varvanin, B. Mayerhofer, and W. PRETTL, Solid State Commun. 97, 827 (1996)

[6] A. Ya. ShUlman, phys. stat. sol. (b) 204, 136 (1997).

[7] S. D. Ganichev, I. N. Yassievich, and W. Prettl, Phys. Solid State 39, 1703 (1997).

[8] A. Ya. Shulman, Proc. Workshop Surface and Interface Optics '99, Sainte Maxime (France), May 4 to 8, 1999; phys. stat. sol. (a) 175, 279 (1999). 